\title{
La flore microbienne du fromage de Roquefort VI. - Les levures
}

\author{
par \\ J. J. DEVOYOD et Dominique SPONEM \\ Station Centrale de Recherches Laitières et de Technologie des Produits Animaux \\ I.N.R.A., Jouy-en-Josas (Yvelines)
}

\section{INTRODUCTION}

Lors d'études précédentes (Devoyod et Bret, 1966 ; Devoyod et al., 1968), nous avions suivi l'évolution de la flore microbienne au cours de plusieurs fabrications traditionnelles de fromage de Roquefort. Nous avions constaté que les levures, peu nombreuses dans le lait de fabrication, se multipliaient rapidement au cours des premières $24 \mathrm{~h}$ qui suivent l'emprésurage. A la surface du fromage, le nombre des levures continuait d'augmenter rapidement jusqu'au salage puis diminuait très nettement au cours du salage. Le nombre des levures augmentait ensuite jusqu'à la mise des fromages sous feuille d'étain, puis, après une légère diminution, il se stabilisait. Au centre du fromage, le nombre des levures augmentait jusqu'au salage; la diminution due à l'action du sel était moins prononcée qu'à la surface. Après une légère augmentation jusqu'à la mise des fromages sous feuille d'étain, le nombre de levures variait peu jusqu'à la commercialisation des fromages.

Kanauchi et al. (1962) ont suivi l'évolution des levures dans le fromage bleu, ils ont constaté que des types différents de levures se trouvaient aux différents stades de la fabrication. Hartley et Jezeski (1954) ont étudié la flore superficielle du fromage bleu, ils ont montré que les levures jouaient un rôle important, après le salage, dans l'implantation de la flore superficielle, composée essentiellement de microcoques et de brevibactéries.

Maxa et Jicinsky (1956) puis Proks et al. (1959) ont montré que des levures du genre Torulopsis, isolées du fromage de Roquefort, contribuaient en partie à la formation de l'arôme des fromages bleus.

Berridge et al. (1953) ont étudié les composants chimiques responsables de l'arôme du fromage de Roquefort et des fromages bleus. 


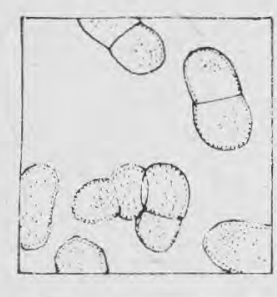

par scissiparité

LEVURES ASCOSPOROGENES

Famille des Endomycetaceae

Reproduction végétative
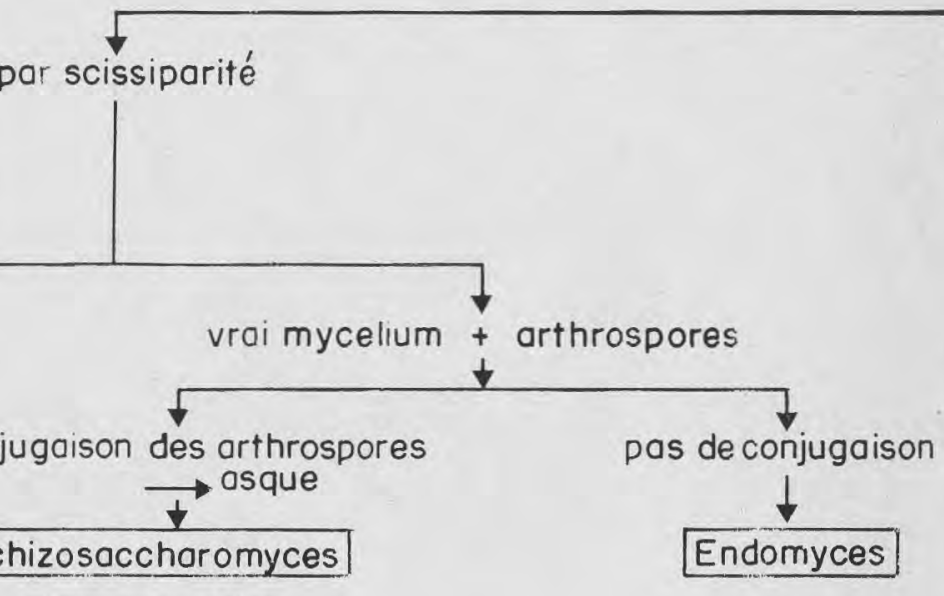

par fission + (ou ) bourgeonnement multilatéral

rai mycelium seul! $\downarrow$

Eremoscus

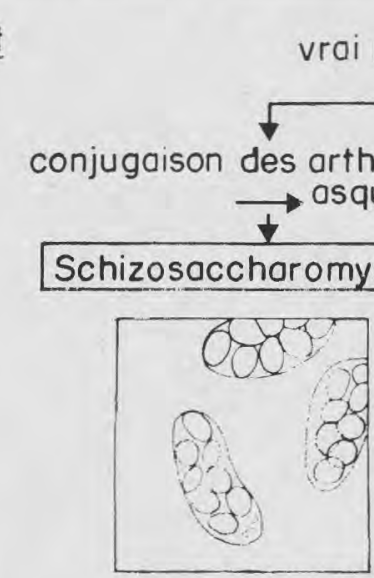

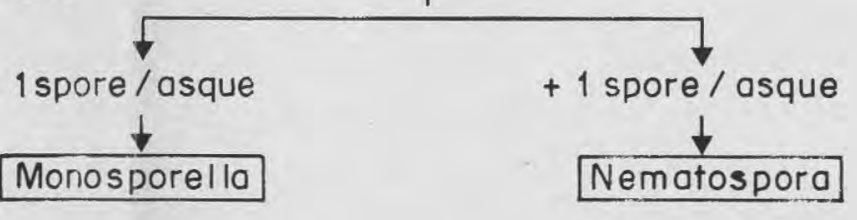

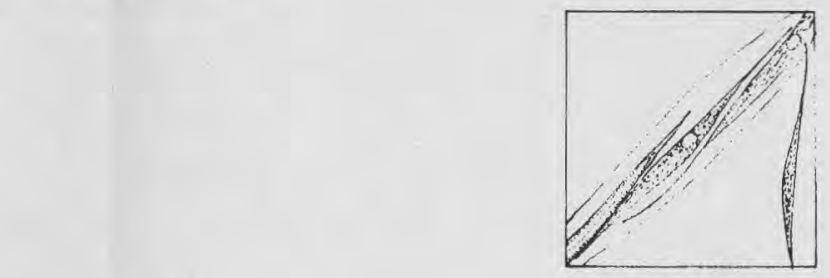

spores fusiformes ou en aiguille spores

pas de pellicule ou pellicule tardive

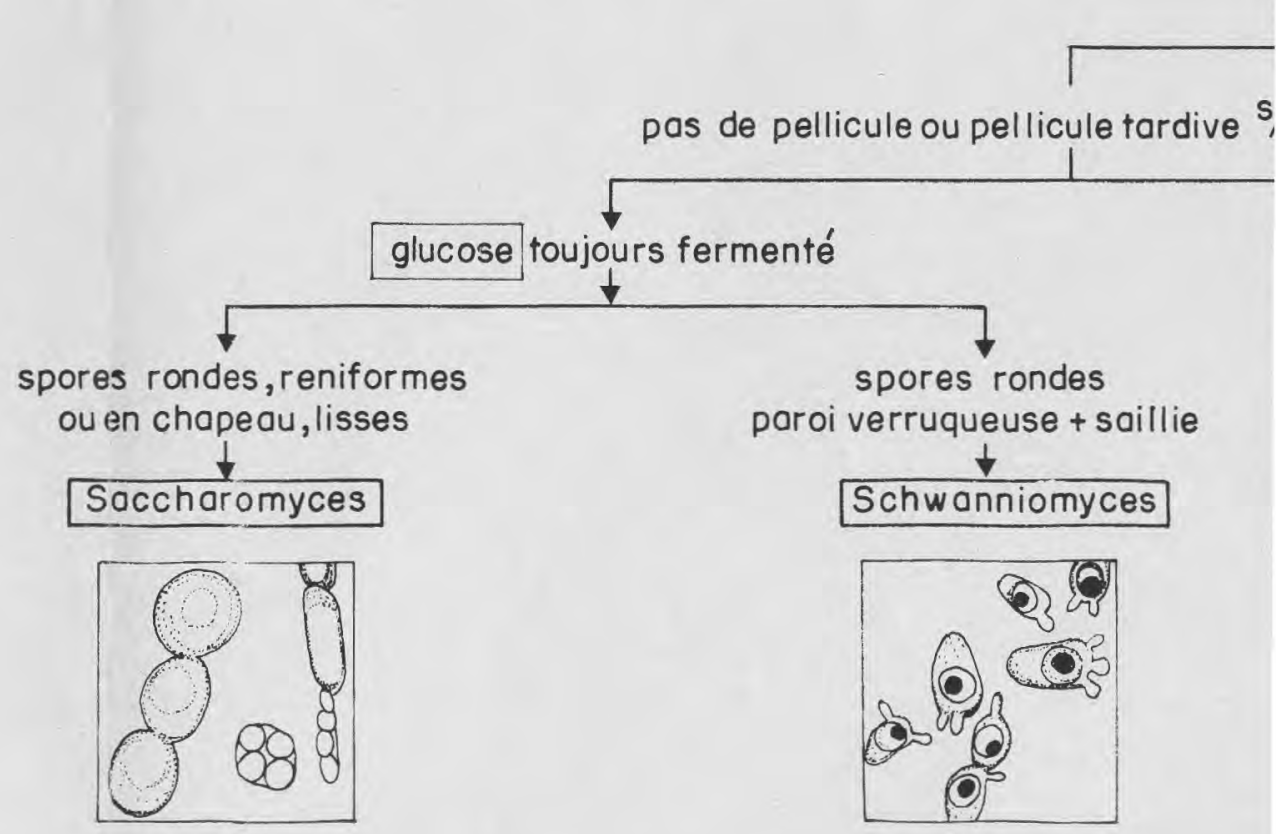

urgeonnan

$\downarrow$
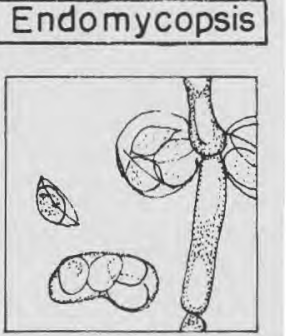
nifusiformes ni en aiguille

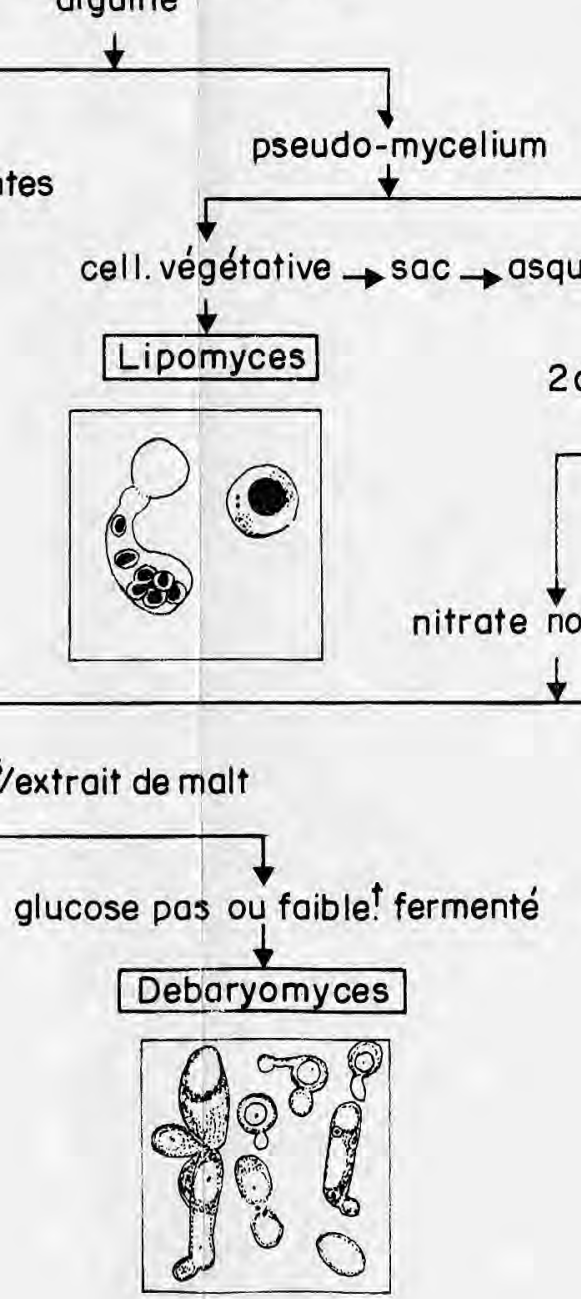

cell. petites

spores rondes $\rightarrow$ chapeau

4 / asque

$$
t
$$

\section{Hanseniaspora}

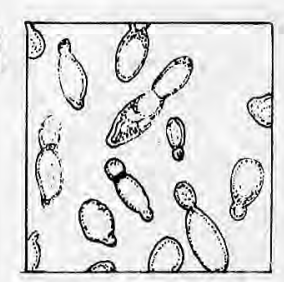

cell. grandes spores rondes_ovales 1.4 / asque

\section{Saccharomycodes}

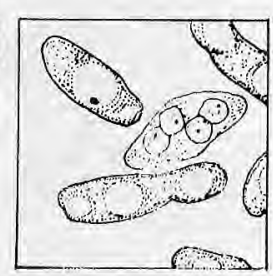

\section{cell. grandes}

spores rondes, paroi verruqueuse 1/asque. conjugaison avant formation de l'asque.

bourgeonnement du zygote$$
\checkmark
$$

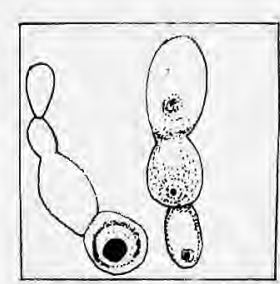


A tous les stades de la fabrication et de l'affinage du fromage de Roquefort nous trouvons des levures en quantité importante ; il est donc permis de penser que ces micro-organismes jouent un rôle non négligeable sur la qualité de ce fromage. Quel est ce rôle ? Actuellement les données que nous possédons sont soit très fragmentaires, soit obtenues par comparaison avec des fromages de même type. C'est la raison pour laquelle il nous a paru intéressant d'étudier les différentes espèces de levures que l'on trouve aux principaux stades de la fabrication, afin de mieux comprendre leur rôle et pouvoir ultérieurement les utiliser à bon escient en fabrication.

Ce travail était achevé quand a été publiée une étude de Galzin et al. (1970) sur la "flore de levure " dans le fromage de Roquefort. Ces auteurs ont montré, en l'absence d'étude taxonomique complète des souches isolées, qu'il existait une grande variété de types biologiques.

\section{Isolement}

\section{MATERIEL ET METHODES}

L'isolement des levures dans les échantillons de lait cru, de caillé au moment de la mise en moules et de fromage aux différents stades de la fabrication et de l'affinage, a été effectué en utilisant une gélose à l'extrait de pomme de terre (Potato Dextrose Agar Difco $\mathrm{n}^{\circ}$ 13) ajustée à $\mathrm{pH}=3,5$ avec de l'acide tartrique. Pour chaque échantillon, 5 à 10 colonies de levures étaient repiquées sur bouillon à l'extrait de malt (Bacto Malt Extract Broth Difco $n^{\circ}$ 113). Après purification, les souches étaient conservées à $+4^{\circ} \mathrm{C}$ sur gélose "mycophile » (B.D. Mérieux) additionnée d'un antifongique : le 2-(4' Thiazolyl) Benzimidazole (concentration finale 0,01 p. 100).

\section{Identificåion}

Pour l'identification des levures nous avons suivi la classification proposée par Lodder et Kreeger-Van Rij (1952). Dans les figures 1 et 2 nous avons indiqué les schémas de différenciation des genres de levures ascosporogènes (Endomycetaceae) et de levures asporogènes (Cryptococcaceae) respectivement.

Les principaux caractères d'identification suivants ont été étudiés :

\section{1) Morphologie}

On effectuait un examen microscopique sur les cultures de levures ayant incubé $72 \mathrm{~h}$ à $22^{\circ} \mathrm{C}$ sur bouillon à l'extrait de malt (Bacto Malt Extract Broth Difco $n^{\circ} 113$ ) à $\mathrm{pH}=4,7$. Une öse de culture était mélangée sur une lame à une goutte de solution lactophénol-acide picrique de composition suivante : acide lactique, $100 \mathrm{ml}$; 
phénol, $100 \mathrm{~g}$; glycérol, $100 \mathrm{ml}$; solution aqueuse saturée d'acide picrique, $100 \mathrm{ml}$ (Harrigan et Mc Cance, 1966).

2) Caractères de la reproduction végétative. Aptitude à former un mycélium

Nous avons utilisé une technique de culture sur lame proche de celle décrite par Wickerham et Duprat (1945). Des lames de verre étaient placées sur des baguettes de verre dans des boîtes de Petri. L'ensemble était stérilisé à sec. Les milieux de culture (Bacto-Potato Dextrose Agar Difco $\mathrm{n}^{\circ} 13$ et Bacto Yeast Morphology Agar Difco $n^{\circ} 393$ ) étaient fondus puis coulés dans une boîte de Petri maintenue à $45^{\circ} \mathrm{C}$ environ. Les lames étaient plongées dans le milieu fondu et replacées sur leur baguette de verre. Après solidification du milieu, les souches de levures étaient inoculées en stries sur les lames. Une lamelle stérile était placée sur les stries d'inoculation. Après 6 j d'incubation à $22^{\circ} \mathrm{C}$, les cultures sur lame étaient examinées au microscope pour rechercher la présence éventuelle de mycélium.

\section{3) Formation d'ascospores}

La propriété de former des ascospores est le caractère le plus important de la classification des levures, permettant de différencier les levures ascosporogènes (Endomycetaceae) des levures asporogènes (Cryptococcaceae).

Le milieu de sporulation utilisé avait la composition suivante : acétate de sodium anhydre 0,5 p. 100 ; gélose 1,5 p. $100 ; \mathrm{pH}=6,5-7,0$ (Fowell, 1952). La présence de spores était recherchée chaque semaine pendant un mois selon la technique de Mc Lung (1943).

\section{4) Formation de pellicule}

La formation de pellicule était observée sur des cultures de levures ayant incubé $72 \mathrm{~h}$ à $22^{\circ} \mathrm{C}$ sur bouillon à l'extrait de malt (Bacto Malt Extract Broth Difco n ${ }^{\circ} 113$ ).

\section{5) Assimilation des sucres. Méthode auxanographique}

Deux techniques sont utilisées dans l'étude de l'assimilation des sucres par les levures : la méthode auxanographique sur milieu gélosé et les essais d'assimilation en tubes. Chacune de ces méthodes présente des avantages et des inconvénients comme l'ont remarqué Barnett et Ingram (1955). Nous avons utilisé la méthode auxanographique ; l'un des inconvénients de cette méthode est le dépôt de sucres non stériles à la surface du milieu ensemencé. Nous avons remédié à cet inconvénient en utilisant des disques de papier imprégnés de sucres et stérilisés (Taxo-disques B.D. Mérieux).

Le milieu utilisé était le Bacto Yeast Nitrogen Base Difco n³92, milieu contenant les vitamines et les oligo-éléments nécessaires à l'assimilation des sucres par les levures (Wickerham et Burton, 1948). $6,7 \mathrm{~g}$ de ce milieu déshydraté étaient dissous dans $100 \mathrm{ml}$ d'eau distillée. Cette solution était stérilisée par filtration sur filtre Seitz 
E.K.S. et conservée à $+4^{\circ} \mathrm{C}$ jusqu'à son utilisation. Dans un tube à essai stérile on mélangeait $5 \mathrm{ml}$ de gélose à $3 \mathrm{p}$. 100 préalablement fondue et maintenue à $47^{\circ} \mathrm{C}, 4 \mathrm{ml}$ d'eau distillée stérile et $1 \mathrm{ml}$ de la solution de Yeast Nitrogen Base maintenus à la même température. Les tubes étaient ensemencés avec $0,1 \mathrm{ml}$ d'une dilution au 1/10 dans du Ringer au 1/4 stérile d'une culture de levures de $48 \mathrm{~h}$ sur bouillon à l'extrait de malt. Le contenu des tubes était versé dans des boîtes de Petri de $60 \mathrm{~mm}$ de diamètre. Après refroidissement, on déposait stérilement à la surface de la gélose un disque de papier imprégné d'un sucre (Taxo-disque B.D. Mérieux). L'incubation des boîtes se faisait à $22^{\circ} \mathrm{C}$. Chaque jour, pendant une semaine, on appréciait la croissance par comparaison avec le milieu ensemencé n'ayant pas reçu de Taxo-disque (boîte témoin). La figure 3 montre, à titre d'exemple, les résultats obtenus avec cette technique. Les sucres suivants ont été essayés : adonitol, arabinose, dulcitol, galactose, glucose, inositol, lactose, maltose, mannitol, mannose, raffinose, rhamnose, saccharose, salicine, sorbitol et xylose.

\section{6) Production d'acide à partir des sucres}

La méthode utilisée était une modification de celle décrite par Sanders et al. (1957). Le milieu employé était le Trypticase Agar Base (B. D. Mérieux) de composition suivante : trypticase, 2 p. 100 ; gélose, 0,35 p. 100 et rouge de phénol, 0,002 p. $100 ; \mathrm{pH}=7,4$. Ce milieu était réparti en tubes de $16 \times 160$ à raison de 12 à $13 \mathrm{ml}$ par tube (tube au $3 / 4$ plein). On introduisait un Taxo-disque à la surface de la gélose à l'aide d'une pince stérile, puis on le faisait pénétrer jusqu'à mi-hauteur du tube à l'aide d'un fil droit. Les levures étaient inoculées en piqûre jusqu'au niveau du Taxo-disque, le fond du tube non inoculé servant de témoin. Les tubes étaient incubés à $22^{\circ} \mathrm{C}$ et les réactions de l'indicateur coloré étaient notées à intervalles réguliers pendant 3 semaines.

\section{7) Fermentation des sucres}

La technique utilisée est celle décrite par Lodder et Kreeger-Van Rij (1952).

\section{8) Assimilation des acides organiques}

Nous avons utilisé la même technique que celle décrite pour l'assimilation des sucres. Après refroidissement des boîtes ensemencées, des disques de papier Durieux $\mathrm{n}^{\circ} 268$ de $9 \mathrm{~mm}$ de diamètre, préalablement stérilisés à l'autoclave, étaient imbibés des solutions d'acides organiques à essayer (par trempage dans des solutions à 1 p. 100) puis déposés à la surface de la gélose. La température d'incubation des boîtes était de $22^{\circ} \mathrm{C}$. Chaque jour, pendant une semaine, on appréciait la croissance par comparaison avec le milieu n'ayant pas reçu de disques. Les acides organiques suivants ont été essayés : acétique, lactique, succinique, formique et citrique. 
Famille des Crypto

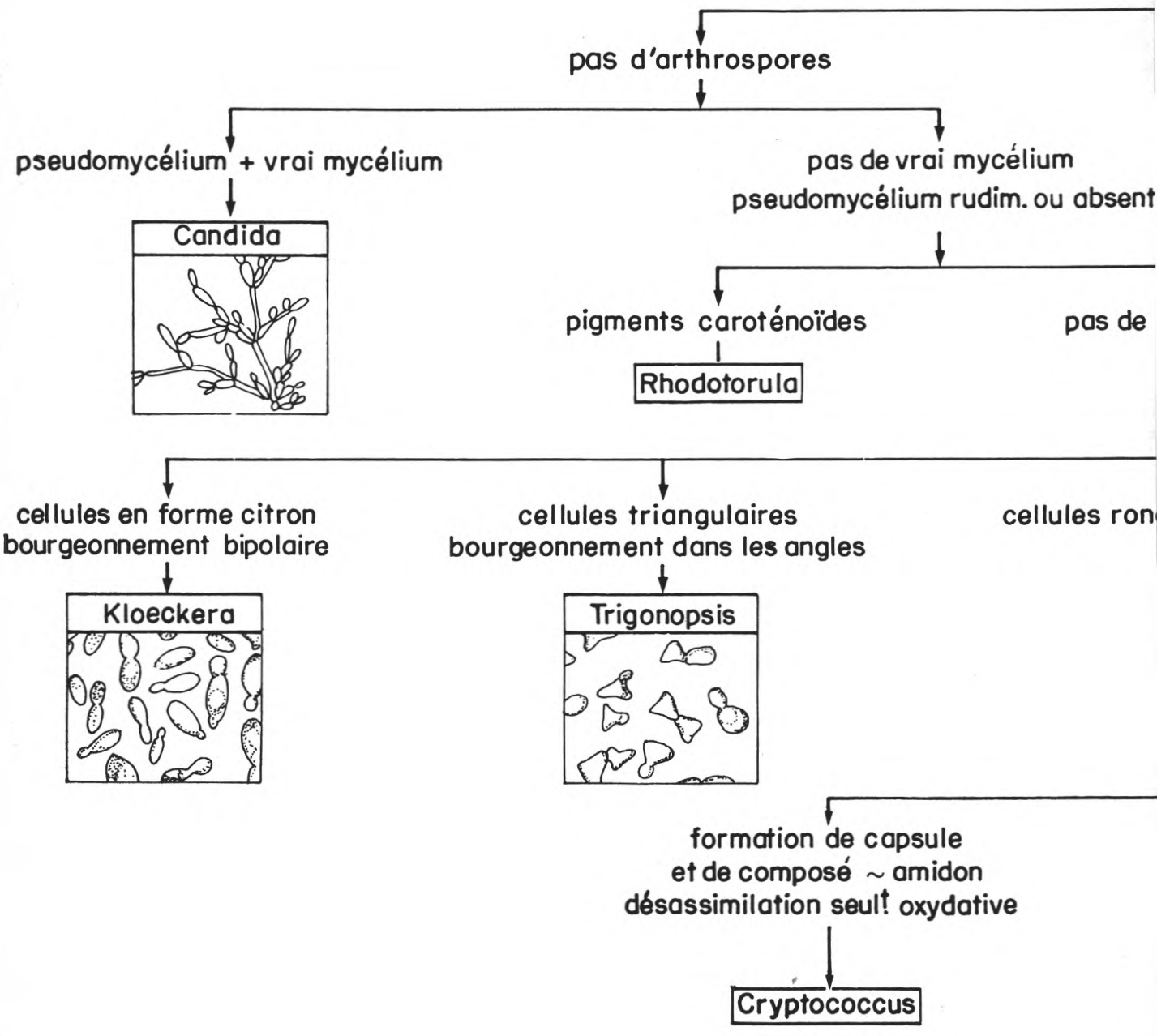

fi

Schéma de différenciation des lev (d'après Lodder 


\section{NES}

ceae

ji mycélium quise morcelle en arthrospores

$t$

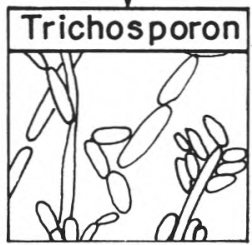

it

Jvales

cellules en forme de bouteille

cellules en ogive bourgeonnement sur la partie large forte prod. acide à partir des sucres
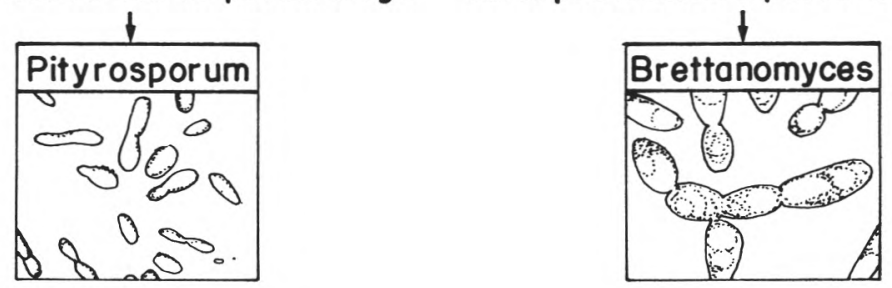

capsule exceptionnelle pas de composé $\sim$ amidon désassimilation oxydative ou oxydative + fermentative Torulopsis 

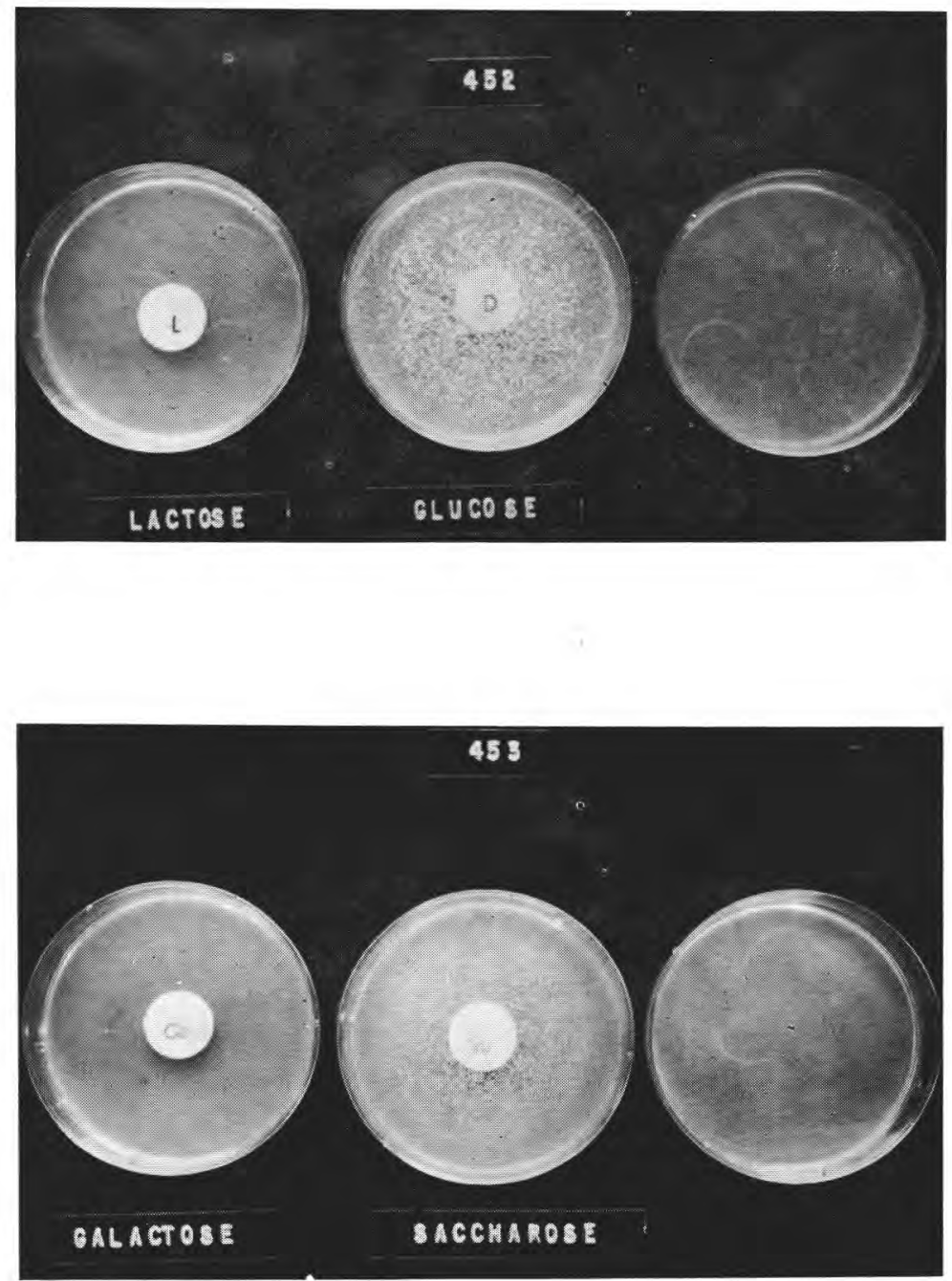

(Photo R. MOURGUES)

fig. 3

Assimilation des sucres par les levures

Méthode auxanographique avec Taxo-disques B. D. Mérieux

A droite: boîte témoin.

A gauche : sucre non assimilé.

Au centre : sucre assimilé. 


\section{9) Assimilation des nitrates}

La recherche de l'assimilation des nitrates est un caractère important d'identification des levures appartenant au genre Hansenula.

Le milieu utilisé était le Bacto Yeast Carbon Base Difco n ${ }^{\circ} 391$. Ce milieu renferme les vitamines reconnues par Wickerham (1946) comme nécessaires pour l'utilisation des composés azotés par certaines levures. $11,7 \mathrm{~g}$ de ce milieu déshydraté était dissous dans $100 \mathrm{ml}$ d'eau distillée. Cette solution était stérilisée par filtration sur filtre Seitz E.K.S. et conservée à $+4^{\circ} \mathrm{C}$ jusqu'à son utilisation. La technique utilisée est la même que celle décrite pour l'assimilation des sucres. Après refroidissement des boîtes ensemencées, des disques de papier filtre Durieux n ${ }^{\circ} 268$ de $9 \mathrm{~mm}$ de diamètre, préalablement stérilisés à l'autoclave, étaient imbibés par trempage dans une solution aqueuse de nitrate de potassium à 1 p. 100, puis déposés à la surface de la gélose. La température d'incubation était de $22^{\circ} \mathrm{C}$. Chaque jour, pendant une semaine, on appréciait la croissance par comparaison avec le milieu ensemencé n'ayant pas reçu de disques.

\section{0) Hydrolyse de l'arbutine}

Le milieu utilisé avait la composition suivante : arbutine, 0,5 p. 100 ; extrait de levure, 1,5 p. 100 ; gélose, 2 p. 100 et citrate ferrique, 0,3 p. 100. Ce milieu était coulé dans des boîtes de Petri et, après solidification, il était ensemencé en surface avec les différentes souches de levures. Les boîtes étaient incubées à $22^{\circ} \mathrm{C}$ et examinées après 2,4 et $6 \mathrm{j}$. Si l'arbutine était hydrolysée, il y avait production d'hydroquinone qui réagissait avec le sel de fer en donnant une coloration brunâtre.

\section{1) Résistance au chlorure de sodium}

Les souches de levures étaient cultivées à $22^{\circ} \mathrm{C}$ pendant $72 \mathrm{~h}$ dans le bouillon à l'extrait de malt contenant $5 ; 7,5 ; 10 ; 12$ et 15 p. 100 de ClNa (concentration finale). Le développement des cultures était apprécié par comparaison avec un tube témoin non ensemencé incubé à la même température (examen de la turbidité).

\section{2) Lipolyse}

La recherche de l'hydrolyse de la tributyrine a été faite selon la méthode conseillée par la "British Standards Institution" (1940).

\section{3) Action sur le lait tournesolé}

Les souches étaient cultivées à $22^{\circ} \mathrm{C}$ et les changements apparus dans le lait tournesolé étaient notés après $48 \mathrm{~h}, 72 \mathrm{~h}, 1$ semaine et 2 semaines d'incubation. 
TABLEAU 1. - Principaux caractères des levures ascosporogènes isolées du fromage de Roquefort

\begin{tabular}{|c|c|c|c|c|}
\hline 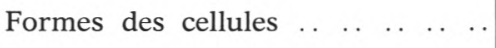 & rondes à ovales & ovales ou longues & petites et rondes & ovales \\
\hline $\begin{array}{llllllll}\text { Bourgeonnement } & . & . & \ldots & \ldots & \ldots & . .\end{array}$ & multilatéral & multilatéral & multilatéral & multilatéral \\
\hline $\begin{array}{llllll}\text { Pouvoir fermentaire } & \ldots & . & \ldots & \ldots & .\end{array}$ & glucose +++ & faible à nul & faible & très net \\
\hline Assimilation des nitrates ...... & - & - & - & + \\
\hline Pellicule sur milieu liquide..... & - & + & + & - \\
\hline Nombre de souches isolées ... .. & 24 & 6 & 9 & 12 \\
\hline $\begin{array}{lllllllllll}\text { Genre } & . . & \ldots & \ldots & \ldots & \ldots & \ldots & \ldots & \ldots & \ldots & . .\end{array}$ & Saccharomyces & Pichia & Debaryomyces & Hansenula \\
\hline
\end{tabular}




\section{RESULTATS}

\section{1) Principaux types de levures}

Quatre-vingt-une souches de levures ont été isolées au cours de la fabrication et de l'affinage du fromage de Roquefort. Parmi ces levures, 51 possédaient la propriété de former des ascospores (elles appartiennent à la famille des Endomycetaceae) et 30 ne possédaient pas cette propriété (elles appartiennent à la famille des Cryptococcaceae).

\section{a) ENDOMYCETACEAE}

Les levures ascosporogènes ont été différentiées en 4 genres : Saccharomyces, Pichia, Debaryomyces et Hansenula (tab. 1).

\section{- Genre Saccharomyces}

Parmi les 24 souches de Saccharomyces, 15 fermentaient le glucose, le galactose, le saccharose, le lactose, mais non le maltose. Elles assimilaient ces 5 sucres et hydrolysaient l'arbutine. On peut considérer ces souches comme appartenant à l'espèce Sacch. lactis (Dombrowski).

Cinq autres souches de Saccharomyces étaient voisines des précédentes, mais elles n'assimilaient pas le maltose et n'hydrolysaient pas l'arbutine. On peut classer ces levures dans l'espèce Sacch. fragilis (Jörgensen).

Enfin 4 souches de Saccharomyces n'ont pu être identifiées avec certitude. Leurs caractères biochimiques étaient proches de ceux de Sacch. lactis, mais la fermentation du lactose était très faible ou absente.

\section{- Genre Pichia}

Les souches de Pichia n'assimilaient que le glucose, 3 d'entre elles fermentaient faiblement le glucose. Toutes ces souches peuvent être classées dans l'espèce $P i$ membranefaciens (Hansen).

\section{- Genre Debaryomyces}

Les souches de Debaryomyces assimilaient le glucose, le galactose, le saccharose, le maltose et le lactose ; elles fermentaient faiblement le glucose et le saccharose. On peut considérer ces souches comme appartenant à l'espèce D. subglobosus (Zach).

\section{- Genre Hansenula}

Les souches d'Hansenula fermentaient le glucose, le saccharose et le maltose, mais non le lactose. La fermentation du galactose était faible. On peut considérer ces souches comme appartenant à l'espèce $H$. anomala (Hansen).

b) CRYPtococcaceae

Parmi les levures asporogènes nous avons identifié 3 genres différents : Torulopsis, Candida et Rhodotorula (tab. 2). 


\section{- Genre Torulopsis}

Parmi les 22 souches de Torulopsis, 9 ne formaient pas de pseudomycélium, fermentaient le glucose, le galactose, le saccharose, le lactose, mais non le maltose. Elles assimilaient ces 5 sucres. Elles n'assimilaient pas le nitrate de potassium et hydrolysaient l'arbutine. On peut classer ces levures dans l'espèce $T$. sphaerica (Hammer et Cordes).

Neuf autres souches de Torulopsis ne fermentaient que peu le glucose, assimilaient le glucose, le galactose, le saccharose, le maltose et le lactose, mais non le nitrate de potassium. Elles hydrolysaient l'arbutine. Ces levures peuvent être considérées comme appartenant à l'espèce $T$. candida (Saito).

Enfin 4 souches de Torulopsis ne fermentaient pas le glucose,

\section{TABLEAU 2}

Principaux caractères des levures asporogènes isolées du fromage de Roquefort

\begin{tabular}{|c|c|c|c|}
\hline Formes des cellules $\ldots \ldots \ldots$ & $\begin{array}{l}\text { rondes } \\
\text { lég. ovales }\end{array}$ & ovales & $\begin{array}{l}\text { rondes } \\
\text { à ovales }\end{array}$ \\
\hline Bourgeonnement $\ldots \ldots \ldots \ldots \ldots$ & multilatéral & multilatéral & multilatéral \\
\hline $\begin{array}{llllll}\text { Pseudo-mycélium } & \ldots & \ldots & \ldots & \ldots\end{array}$ & - & + & - \\
\hline Pouvoir fermentaire $\ldots \ldots \ldots$ & net & net & absent \\
\hline $\begin{array}{lllllllll}\text { Pigment } & \ldots & \ldots & \ldots & \ldots & \ldots & \ldots & \ldots & \ldots\end{array}$ & - & - & rose à rouge \\
\hline $\begin{array}{l}\text { Formation de composés type ami- } \\
\text { don } \ldots \ldots\end{array}$ & - & - & - \\
\hline Nombre de souches isolées ... . . & 22 & 4 & 4 \\
\hline Genre $\ldots \ldots \ldots \ldots \ldots \ldots \ldots$ & Torulopsis & Candida & Rhodotorula \\
\hline
\end{tabular}

assimilaient le glucose, le galactose, le saccharose, le maltose mais non le lactose. On peut considérer ces souches comme appartenant à l'espèce $T$. famata (Harrison).

\section{- Genre Candida}

Les caractères des souches de Candida permettent de les classer dans l'espèce $C$. lipolytica (Harrison), bien que la digestion du lait ne soit pas très fortement marquée.

\section{2) Evolution des levures dans le fromage de Roquefort}

La figure 4 rappelle l'évolution des levures au cours d'une fabrication traditionnelle de fromage de Roquefort (Devoyod et al., 1968). 


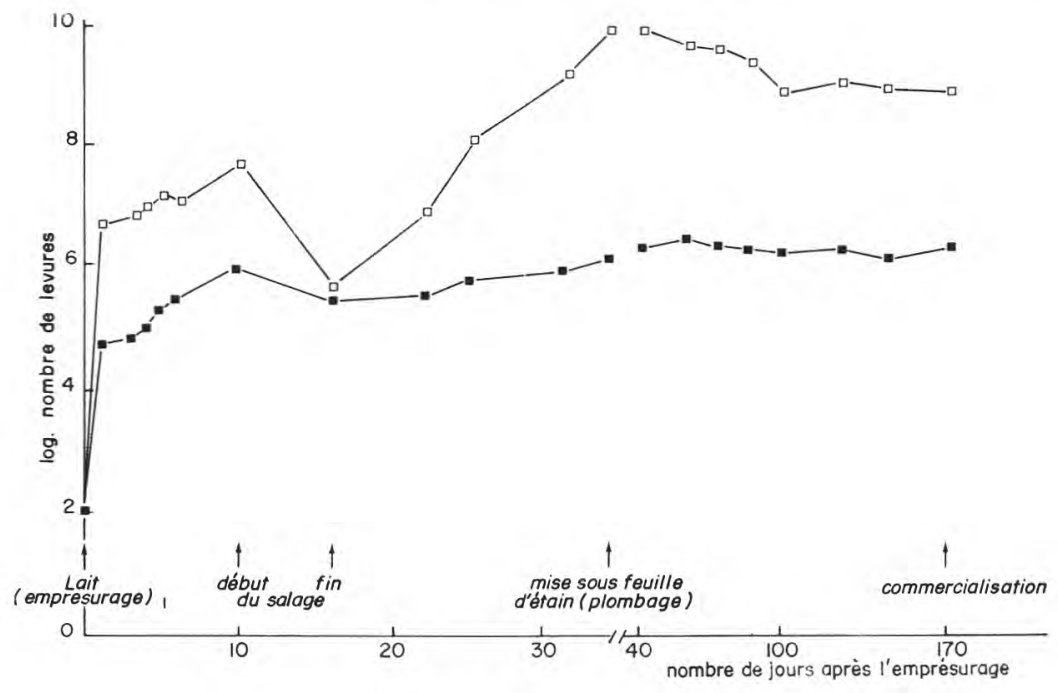

fig. 4

Evolution des levures au cours de la fabrication et de l'affinage du fromage de Roquefort

-

$\square-\square$ surface du fromage

Dans le tableau 3 nous avons porté les types de levures isolées aux principaux stades de la fabrication du fromage de Roquefort ; dans ce tableau nous avons indiqué également la résistance au chlorure de sodium de ces levures ainsi que leur pouvoir de fermenter le lactose.

Les levures isolées avant salage sont principalement des espèces fermentant le lactose (Sacch. lactis, Sacch. fragilis et T. sphaerica).

La première constatation que l'on peut faire est que ces levures sont biologiquement et physiologiquement très proches les unes des autres. Sacch. lactis et Sacch. fragilis sont des espèces voisines; quant à $T$. sphaerica, elle est considérée comme étant la forme imparfaite de Sacch. lactis (Lodder et Kreeger-Van Rij., 1952). Ces levures sont peu résistantes au sel. Par chromatographie en phase gazeuse, nous avons mis en évidence la production d'éthanol et d'acétaldéhyde dans les cultures de Sacch. lactis, Sacch. fragilis et T. sphaerica. Au cours de notre étude, nous avons constaté que les colonies de certaines souches de Sacch. lactis, colonies blanches obtenues sur gélose à l'extrait de pomme de terre, prenaient une teinte rougeâtre lorsqu'elles étaient en contact avec le mycélium de Penicillium roqueforti. Cette observation est à rapprocher de celle faite par Wickerman et Burton (1956) qui 
TABLEAU 3. - Evolution qualitative des levures dans le fromage de Roquefort

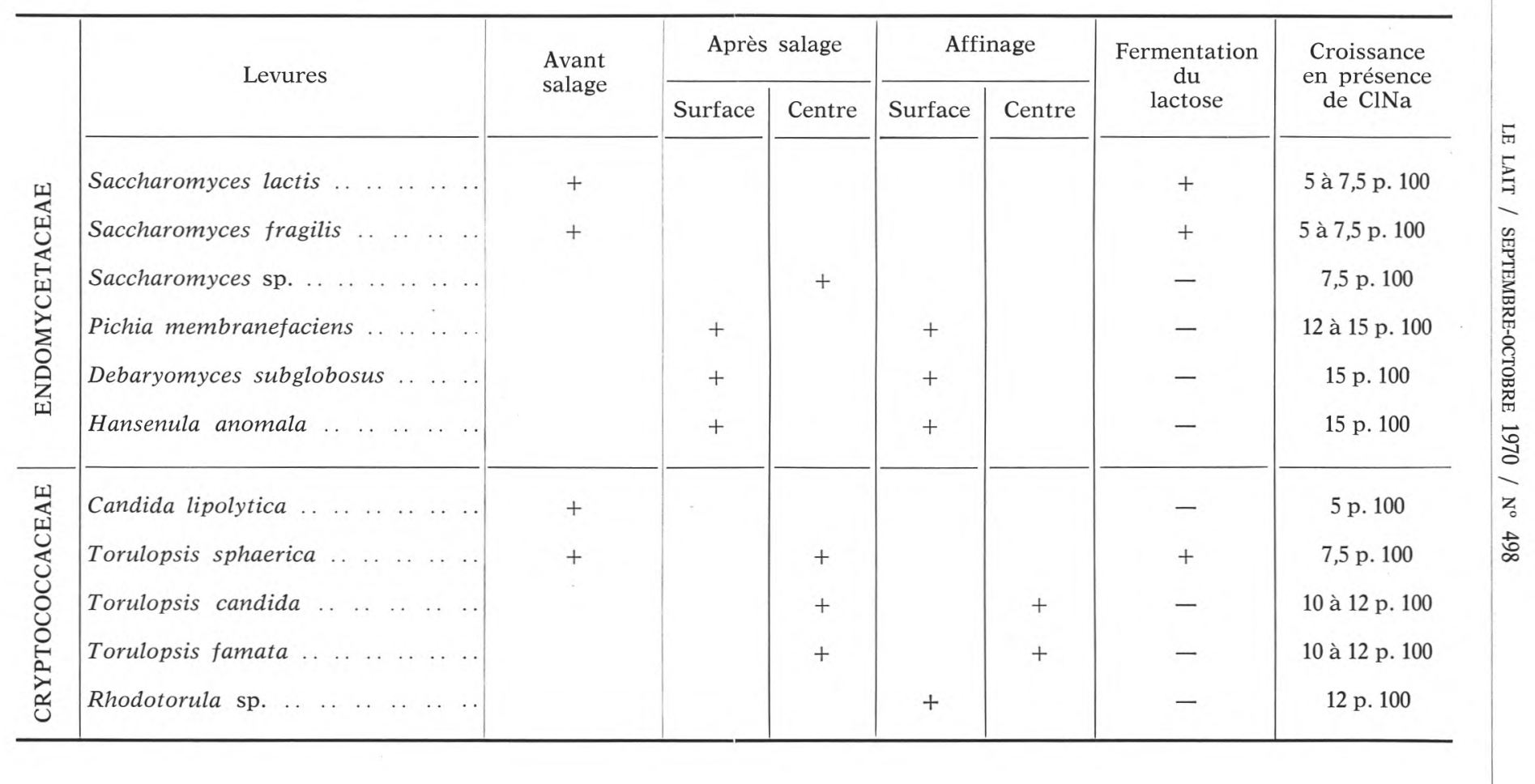


ont montré que plusieurs levures fermentant le lactose produisaient un pigment semblable à la pulchérimine.

$\mathrm{Au}$ centre du fromage après salage et au cours de l'affinage, nous trouvons essentiellement des levures asporogènes appartenant au genre Torulopsis. Ces levures sont plus résistantes au sel que les Saccharomyces.

A la surface du fromage, nous trouvons des levures différentes de celles isolées au centre du fromage. Ce sont surtout des levures sporogènes ( $H$. anomala, Pi. membranefaciens et $D$. subglobosus). Ces levures ne fermentent pas le lactose et sont très résistantes au chlorure de sodium.

Nos résultats montrent que le salage du fromage a un rôle sélectif sur les levures.

\section{DISCUSSION}

\section{1) Les levures isolées avant salage}

a) LES LEVURES FERMENTANT LE LACTOSE

Ces levures ne sont pas spécifiques des fromages fabriqués à partir de lait de brebis, elles ont été en effet souvent mises en évidence dans les produits laitiers (Dombrowski, 1910 ; Saccheti, 1932 et 1933 ; Guitonneau et al., 1939 ; Capriotti, 1957 ; Picci, 1960 ; Cooke et Brazis, 1968 ; Ghoniem, 1968 ; Nakanishi et Arai, 1968 et 1969). Le plus souvent ces levures ont été étudiées en raison des défauts qu'elles provoquent dans les fromages (gonflements) par suite d'une production trop importante de gaz (Stocker, 1954 ; Sahli, 1960).

Parmi ces levures fermentant le lactose, Sacch. fragilis a été plus particulièrement étudiée en vue de sa culture sur le lactosérum (sous-produit de la fromagerie) et de son utilisation comme levurealiment (Porges et al., 1951 ; Wasserman, 1960 et 1961 ; Wasserman et al., 1958, 1959, 1960 et 1961).

Pour que le Penicillium puisse se développer dans le fromage de Roquefort et lui donner son aspect " persillé " caractéristique, il est nécessaire que le caillé soit " ouvert ". Cette ouverture, que l'on observe au cours des premières $48 \mathrm{~h}$ qui suivent l'emprésurage, est due à une production de gaz, essentiellement de $\mathrm{CO}_{2}$ (Bret, 1965). Or, l'un des produits de la fermentation du lactose par les levures est le $\mathrm{CO}_{2}$. Le fait que les levures fermentant le lactose se multiplient au cours des premières $24 \mathrm{~h}$ qui suivent l'emprésurage, permet de supposer qu'elles jouent un rôle dans l'ouverture du caillé. Nous avons constaté en effet que lorsqu'on ajoute une culture de Sacch. lactis sur lactosérum à du lait pasteurisé de brebis (la pasteurisation ayant pour but dans ce cas d'éliminer les levures du lait), les fromages fabriqués avec ce lait ensemencé présentent après $48 \mathrm{~h}$ une ouver- 
ture correcte, tandis que les fromages témoins, fabriqués avec le lait pasteurisé non ensemencé en levures fermentant le lactose, restent peu « ouverts ».

Dans d'autres types de fromages fabriqués à partir de lait cru (pâtes molles, pâtes pressées), nous trouvons également des levures fermentant le lactose ; or ces fromages, normalement, ne présentent pas d'ouverture. De plus, lorsque ces fromages renferment de grandes quantités de levures fermentant le lactose, la production de $\mathrm{CO}_{z}$ est telle que ces fromages " gonflent ". Dans le fromage de Roquefort, tout se passe comme si un équilibre s'établissait entre les levures fermentant le lactose et divers facteurs (microbiens, physicochimiques, etc.) de telle sorte que la production de $\mathrm{CO}_{2}$ par les levures soit limitée. English (1953) a montré que, dans des cultures de levures, la fermentation devenait appréciable lorsque le nombre de ces micro-organismes était compris entre $10^{\mathrm{a}}$ et $10^{5}-10^{\mathrm{b}}$ cellules $/ \mathrm{ml}$. Or, si nous regardons la courbe d'évolution des levures au centre du fromage (fig. 4), nous constatons une très forte croissance des levures au cours des premières $24 \mathrm{~h}$ puis un palier qui se situe en dessous de $10^{5}$ cellules/g, montrant ainsi, qu'à ce stade, la multiplication des levures a été stoppée. Les facteurs qui ont une action sur 'cette « stabilisation " des levures à un niveau optimum sont actuellement en cours d'étude; les résultats de ces travaux feront l'objet d'une publication ultérieure. Il semblerait que la flore lactique joue un rôle important (Stocker, 1954).

Actuellement, on peut admettre que l'ouverture du fromage de Roquefort est due essentiellement à l'action de deux types de microorganismes ; d'une part les Leuconostoc qui seuls sont de faibles producteurs de $\mathrm{CO}_{2}$ mais qui donnent d'excellents résultats lorsque cette production de $\mathrm{CO}_{2}$ est stimulée (Devoyod et Muller, 1969) et d'autre part les levures fermentant le lactose qui sont de fortes productrices de $\mathrm{CO}_{2}$ et qui donnent aussi d'excellents résultats lorsqu'elles sont maintenues à un niveau convenable.

Nous avons vu que les levures fermentant le lactose produisaient de l'éthanol. Or, Maxa et Jicinsky (1956) estiment que la production d'éthanol, permettant la formation d'esters d'acides gras, a une action favorable sur le goût du fromage bleu. Il est donc permis de penser que ces levures ont une action sur le goût du fromage. Comme pour la production de $\mathrm{CO}_{2}$, il semble qu'une production trop importante d'éthanol soit défavorable. En effet, lors d'expérimentations effectuées en ensemençant du lait avec une quantité importante de Sacch. lactis $\left(10^{7}\right.$ cellules $\left./ \mathrm{ml}\right)$, les fromage obtenus avaient, en fin d'affinage, un goût d'alcool très prononcé.

Sacch. lactis, Sacch. fragilis et T. sphaerica produisent également de l'acétaldéhyde. Or Morgan et Andersson (1956) pensent que l'acétaldéhyde est un constituant du goût des fromages bleus. Ils estiment par contre que l'acétaldéhyde est produite par Streptococcus lactis et certaines de ses variétés (Zuraw et Morgan, 1951; Jackson et 
Morgan, 1954). On peut penser que les levures fermentant le lactose contribuent soit seules, soit avec les streptocoques lactiques, à la saveur typique du fromage de Roquefort.

\section{b) LES LEVURES DU GENRE CANDIDA}

Nos résultats ont montré que $C$. lipolytica était présente dans le fromage de Roquefort avant salage. Or les travaux de Parmelee et Nelson (1949 a) prouvent que l'addition d'une culture de C. lipolytica au lait de fabrication des fromages bleus a une action bénéfique sur l'arôme de ces fromages et augmente la teneur en acides gras volatils de ces fromages. Ces mêmes auteurs (1949 b) ont montré de plus que l'addition au lait pasteurisé d'acides gras de faible poids moléculaire améliore l'arôme du fromage. Certes ces résultats ont été obtenus avec des laits pasteurisés, c'est-à-dire avec des laits dont la lipase naturelle avait été détruite, mais il n'est pas interdit de penser que, dans les laits crus, $C$. lipolytica puisse jouer un rôle dans la formation de l'arôme du fromage.

\section{2) Les levures de la flore superficielle}

Dans le fromage de Roquefort, les levures qui se développent à la surface après le salage et au cours de l'affinage sont différentes de celles que l'on trouve au centre du fromage. Des observations semblables ont été faites pour le fromage bleu par d'autres auteurs (Hartley et Jezeski, 1954 ; Kanauchi et al., 1962). Il est vraisemblable que les levures qui se développent en surface du fromage ont une origine " externe " provenant soit de l'atmosphère des saloirs et des caves, soit du sel. Di Menna (1955) a isolé Debaryomyces subglobosus et plusieurs espèces de Rhodotorula à partir de l'atmosphère. Connell et Skinner (1953) de leur côté ont montré que l'atmosphère renfermait de nombreux genres de levures, notamment Rhodotorula. Lors d'analyses microbiologiques de sels marins, nous avons nous-mêmes dénombré des levures dans plusieurs échantillons (Devoyod, 1966 ; résultats non publiés). Phaff et al. (1952) ont trouvé qu'il existait une grande similitude entre les levures de l'eau de mer et celles isolées d'aliments d'origine marine. Lors d'une étude précédente (Devoyod, 1969) nous avons montré nous-mêmes que le sel marin utilisé pour le salage du fromage jouait un rôle important dans l'ensemencement microbien de la surface du fromage. Dans le cas des levures, qui sont des micro-organismes acidophiles, le $\mathrm{pH}$ du fromage ne joue pas un rôle sélectif comme dans le cas des microcoques par exemple. Il est vraisemblable que la forte teneur en chlorure de sodium (15 à $20 \mathrm{~g}$ de $\mathrm{CINa}$ p. 100 de la phase aqueuse, Devoyod et al., 1968) que l'on constate à la surface du fromage de Roquefort après le salage a une action bénéfique sur le développement des levures appartenant aux genres Hansenula, Pichia et Debaryomyces. Etchells et al. (1953) ont montré en effet que le développement des levures était meilleur dans des saumures contenant 10 
à 15 p. 100 de ClNa que dans des saumures contenant 5 p. 100 de CINa, parce que les levures les plus halo-résistantes (Hansenula, Pichia et Debaryomyces entre autres) étaient favorisées dans leur compétition avec les autres micro-organismes. Nous avons constaté que, lorsque l'acidification du fromage était insuffisante, lorsque les fromages étaient moins salés ou salés avec des sels très contaminés en microcoques $(70000 / \mathrm{g}$ par exemple), il se produisait souvent à la surface du fromage une multiplication importante, bien que localisée, de microcoques caséolytiques. Cette multiplication anormale avait pour résultat l'apparition de défauts qui se présentaient sous forme de taches circulaires dont l'aspect leur a fait donner le nom d' " auréoles ". Les analyses microbiologiques ont toujours montré que la principale différence entre les zones "saines " et les zones "tarées " était une inversion du rapport entre le nombre de levures et le nombre de microcoques.

Nos résultats ont montré que les levures qui se développaient à la surface du fromage après le salage et au cours de l'affinage étaient principalement $H$. anomala, $P i$. membranefaciens et $D$. subglobosus. Dans nos études sur la flore microbienne du fromage de Roquefort, nous avons suivi des fabrications traditionnelles dans le périmètre proche de Roquefort. Les fromages étaient fabriqués dans les laiteries et le salage était effectué dans les caves de Roquefort. Par contre, pour les laiteries éloignées, telles que celles que l'on rencontre dans les Pyrénées ou en Corse par exemple, le salage est effectué sur place et les fromages salés sont ensuite affinés dans les caves de Roquefort. On conçoit aisément que l'on puisse trouver d'autres espèces de levures que celles que nous avons isolées et ce à cause de la différence de lot de sel ou de la différence de la flore atmosphérique des saloirs. Ainsi, lors d'analyses microbiologiques effectuées sur des fromages frabriqués et salés dans une laiterie Corse, nous avons trouvé, dans les échantillons prélevés à la surface, une prédominance de levures pigmentées, roses à rougeâtres, qui étaient vraisemblablement des Rhodotorula (Devoyod, 1966 ; résultats non publiés).

Le développement des levures à la surface du fromage après le salage n'est pas un caractère propre au fromage de Roquefort ; on constate ce développement dans d'autres fromage (Kelly, 1937 ; Macy et Erekson, 1937 ; Langhus et al., 1945 ; Hartley et Jezeski, 1954; Kanaushi et al., 1962). Il est couramment admis que le rôle essentiel de ces levures est de métaboliser l'acide lactique, ce qui permet le développement ultérieur des micro-organismes acido-sensibles (microcoques et brévibactéries). A la surface du fromage de Roquefort, on constate bien en effet dans les jours qui suivent le salage, d'abord une multiplication des levures, puis une multiplication des microcoques. Toutefois au cours de cette période les valeurs de $\mathrm{pH}$ restent inférieures à 5,6 - 5,8 (Devoyod et Bret, 1966 ; Devoyod et al., 1968) ; or, les microcoques seuls se développent mal à ces pH. 
Il est vraisemblable que les levures apportent des substances stimulantes aux microcoques, comme cela a été montré dans le cas de Brevibacterium linens (Purko et al., 1945). Dans les fromages bleus, les levures sont généralement reconnues comme intermédiaires dans l'établissement de la flore superficielle; dans le cas du fromage de Roquefort, il semble bien qu'elles font parties de la flore superficielle puisqu'on les trouve en effet en flore dominante, avec les microcoques, à la surface du fromage tout au long de la période d'affinage.

\section{3) Les levures isolées au centre du fromage après salage et au cours de l'affinage}

Nos résultats ont montré que les levures isolées au centre du fromage après salage et au cours de l'affinage appartiennent principalement au genre Torulopsis. Les espèces isolées correspondent à celles étudiées par Proks et al. (1959). Ces auteurs ont montré que $T$. sphaerica (que nous avons aussi trouvé avant salage) avait une action stimulante vis-à-vis de la production, par Penicillium roqueforti, de méthyl-cétones, composés importants de l'arôme typique du fromage de Roquefort (Berridge et al., 1953 ; Morgan et Anderson, 1956). T. candida possédait également, mais à un degré moindre, un pouvoir stimulant vis-à-vis de Penicillium roqueforti. Quant à $T$. famata elle ne présentait aucune action sur la production d'arôme par la moisissure bleue.

Le fait que les Torulopsis soient abondantes dans le fromage de Roquefort (de l'ordre de $10^{7} / \mathrm{g}$ ) permet d'affirmer, qu'à côté de Penicillium roqueforti, comme dans le cas du fromage tchèque Niva, elles jouent un rôle dans l'obtention de la saveur typique du fromage de Roquefort.

\section{$R$ é $\mathbf{s}$ u é}

Quatre-vingt-une souches de levures ont été isolées du fromage de Roquefort. Les levures que l'on trouve au centre du fromage avant le salage sont essentiellement des levures fermentant le lactose (Saccharomyces lactis, Saccharomyces fragilis et Torulopsis sphaerica). Les levures isolées à la surface du fromage après le salage et au cours de l'affinage sont très résistantes au sel et appartiennent aux genres Pichia, Hansenula, Debaryomyces et Rhodotorula. Les levures que l'on trouve au centre du fromage au cours de l'affinage sont asporogènes et appartiennent au genre Torulopsis. Le rôle possible des levures dans le fromage de Roquefort est discuté. 


\section{Remerciements}

Nous remercions vivement $M$. Galzy, Maître de Conférence à l'E.N.S.A. de Montpellier de nous avoir transmis des levures types de sa collection personnelle.

Nous remercions $M$. Bret, ancien Directeur du Laboratoire de la Société des Caves de Roquefort, pour l'aide qu'il nous a apporté dans la réalisation de notre étude.

Nos remerciements vont également à MM. Auclair, Hermier et Raibaud pour leurs suggestions et leurs critiques qui ont été pour nous une aide précieuse dans l'élaboration et la présentation de ce travait.

Nous remercions $M$. Goto de l'aide qu'il nous a apporté en traduisant les publications japonaises et Mme Tramasaygues pour la réalisation des schémas de ce texte.

Microbial flora of Roquefort cheese

VI. Yeasts

\section{S u m m a ry}

81 strains of yeasts were isolated from Roquefort cheese. The yeasts found in the inner part of the cheese before salting are mainly lactose-fermenting yeasts (Saccharomyces lactis, Saccharomyces fragilis and Torulopsis sphaerica). The yeasts isolated from the surface of the cheese after salting and during the ripening period are very salt-resisting yeasts and belong to the genus Pichia, Hansenula, Debaryomyces and Rhodotorula. The yeasts found in the inner part of the cheese during the ripening period are asporogenous yeasts and belong to the genus Torulopsis. The possible role played by yeasts in Roquefort cheese is discussed.

Reçu pour publication en juin 1970.

\section{Références bibliographiques}

[1] Barnett (J. A.) and Ingram (M.) (1955). - Technique in the study of yeast assimilation reactions. J. appl. Bact., 18, 131.

[2] Berridge (N. J.), Hiscox (E. R.) and Zielenska (M.) (1953). - Some flavour constituents of english cheese. Int. Dairy Congr., The Hague, 2, 587.

[3] BRET (G.) (1965). - Communication personnelle.

[4] British Standards Institution (1940). - Stand. Specif. No 895 . Methods for the microbiological examination of butter.

[5] CapriotTi (A.) (1957). - I lieviti nella muturazione dei formaggio. I Nota : Formaggio Limburgo. Latte, 31, 235. 
[6] Connell (G. H.) and Skinner (C. E.) (1953). - The external surface of the human body as a habitat for non-fermenting non-pigmented yeasts. J. Bact., 66,627 .

[7] COOKE (W. B.) and Brazis (A. R.) (1968). - Occurrence of molds and yeasts in dairy products. Mycopathol. Mycol. Appl., 35, 281.

[8] Devoyod (J. J.) (1969). - La flore microbienne du fromage de Roquefort. II. Staphylocoques et microcoques. Le Lait, 481-482, 1.

[9] Devoyod (J. J.) et BRET (G.) (1966). - Evolution de la flore microbienne au cours de la fabrication et de l'affinage du fromage de Roquefort. $X V I I^{\mathrm{e}}$ Cong. Int. de Laiterie, D-2, 585.

[10] Devoyod (J. J.), Bret (G.) et Auclair (J. E.) (1968). - La flore microbienne du fromage de Roquefort. I. Son évolution au cours de la fabrication et de l'affinage. Le Lait, 479-480, 613.

[11] Devoyod (J. J.) et Mul.LeR (M.) (1969). - La flore microbienne du fromage de Roquefort. III. Les streptocoques lactiques et les Leuconostoc. Influence de différents micro-organismes de contamination. Le Lait, 487, 1.

[12] Di Menna (M. E.) (1955). Cité par Ingram (M.) (1958), - Yeasts in food spoliage ; dans The chemistry and biology of yeasts. Academic Press Inc. New-York, p. 627.

[13] Dombrowski (W.) (1910). - Die Hefen in Milch und Milchprodukten. Beitrag zur Kenntnis der Mikroflora der Milch und der Milchprodukte. Zbl. Bakteriol., Abt. 2, 28, 345.

[14] ENGLish (M. P.) (1953). - The fermentation of malt extract by an osmophilic yeast. J. Gen. Microbiol., 9, 15.

[15] Etchells (J. L.), Bell (T. A.) and Jones (I. D.) (1953). Cité par IngRam (M.) (1958). - Yeasts in food spoliage ; dans The chemistry and biology of yeasts. Academic Press Inc. New-York, p. 625.

[16] Fowell (R. R.) (1952). - Sodium acetate agar as a sporulation medium for yeast. Nature, Lond., 170, 578.

[17] Galzin (M.), Galzy (P.) et BRET (G.) (1970). - Etude de la flore de levure dans le fromage de Roquefort. Le Lait, 491-492, 1.

[18] Ghoniem (N. A.) (1968). - Incidence of yeasts other than Candida species in Damietta cheese. Milchwissenschaft, 23, 482.

[19] Guittonneau (G.), Keilling (J.) et Delaval (H.) (1939). - Les formes de levures dans la flore superficielle des fromages de Camembert. Le Lait, 19, 338 .

[20] HaRrigan (W. F.) and Mc CANCE (M. E.) (1966). - Laboratory methods in microbiology. Academic Press, London and New-York, p. 84.

[21] Hartley (C. B.) and Jezeski (J. J.) (1954). - The microflora of blue cheese slime. J. Dairy Sci., 37, 436.

[22] JaCKSON (H. W.) and Morgan (M. E.) (1954). - Identification and origin of the malty aroma substance from milk cultures of Streptococcus lactis var. maltigenes. J. Dairy Sci., 37, 1316.

[23] Kanauchi (T.), Yoshioka (Y.) and Намамото (M.) (1962). - Microbial studies on blue veined cheese. I. Microflora of blue veined cheese in ripening period. Jap. J. Zootech. Sci., 32, 104.

[24] Kelly (C. D.) (1937). - The microbial flora on the surface of Limburger cheese. J. Dairy Sci., 20, 239.

[25] Langhus (W. L.), Price (W. V.), Sommer (H. H.) and Frazier (W. C.) (1945). The "smear " of brick cheese and its relation to flavor development. J. Dairy Sci., 28, 827.

[26] Lodder (J.) and Kreeger-VAN RiJ (N. J. W.) (1952), - The yeasts. A taxonomic study. North Holland Publishing Company, Amsterdam. 
[27] Mc Lung (L. S.) (1943). - On the staining of yeast spores Science, 98, 159.

[28] MACr (H.) and EREKSON (J.A.) (1937). - Microflora of cheese slime. J. Dairy Sci., 20, 464.

[29] MAXA (V.) and Jicinsky (V.) (1956). - A study of some asporogenous yeasts in view of their application in the manufacture of Roquefort cheese. Int. Dairy Congr., 2, 345.

[30] Morgan (M. E.) and Anderson (E. O.) (1956). - The neutral carbonyl compounds in blue-mold type cheese. J. Dairy Sci., 39, 253.

[31] NaKanishi (T.) and ARAI (I.) (1968). - Studies on lactose fermenting yeasts. I. Isolation and identification of lactose fermenting yeasts from raw milk. (Part I). Jap. J. Dairy Sci., 17, A 152.

[32] NaKanishi (T.) and Arai (I.) (1969). - Studies on lactose fermenting yeasts. II. Isolation and identification of lactose fermenting yeasts from raw milk. (Part II). Jap. J. Dairy Sci., 18, A 25.

[33] Parmelee (C. E.) and Nelson (F. E.) (1949 a), - The use of Candida lipolytica in the manufacture of blue cheese from pasteurized homogenized milk. J. Dairy Sci., 32, 993.

[34] Parmelee (C. E.) and Nelson (F. E.) (1949 b). - The relation of chemical analyses to the flavor scores of blue cheese made from pasteurized homogenized milk. J. Dairy Sci., 32, 1007.

[35] Phaff (H. J.), Mrak (E. M.) and Williams (O. B.) (1952), - Yeasts isolated from shrimp. Mycologia, 44, 431 .

[36] PIcci (G.) (1960). - Sopra i lieviti presenti in alcuni formaggi greci. Ann. Fac. Agric. Pisa, 21, 149.

[37] Porges (N. I.), Pepinsky (J. B.) and Jasewicz (L.) (1951). - Feed yeast from dairy by-products. J. Dairy Sci., 34, 615.

[38] Proks (J.), Dolezalek (J.) and Pech (Z.) (1959). - A study of the coaction of the yeasts of the genus Torulopsis on the formation of the methyl ketones in the cheese of Roquefort type. Int. Dairy Congr., 2, 345.

[39] Purko (M.), Nelson (W. O.) and Wood (W. A.) (1951). - The associative growth between certain yeasts and Bacterium linens. J. Dairy Sci., 34, 699.

[40] Sacchetti (M.) (1932). - Contributo alla conoscenza della flora microbica di alcuni formaggi italiani. $1^{\circ}$ Nota. Arch. Mikrobiol., 3, 650.

[41] SacchetTi (M.) (1933). - Contributo alla conoscenza della flora microbica di alcuni formaggi italiani. $2^{\circ}$ Nota. Arch. Mikrobiol., 4, 427.

[42] SAHLi (K. W.) (1960). - Uber die Möglichkeit einer Fehlgärung oder Blähung von Hartkäse durch Lactose vergärende Hefen (Milchzuckerhefen). Schweiz. Milchztg., 86, Wissenchaftliche Beilage, 73, 581.

[43] Sanders (A. C.) Faber (J. E.) and CooK (T. M.) (1957). - A rapid method for the characterization of enteric pathogen using paper discs. Appl. Microbiol, 5,36 .

[44] STOCKeR (W.) (1954). - Hefen als Käsebläher - Milchsäurebakterien als Antibionten. Dtsch. Molk. Ztg., 75, 131.

[45] Wasserman (A. E.) (1960), - Whey utilization. IV. Avaibility of whey nitrogen for the growth of Saccharomyces fragilis.

[46] Wasserman (A. E.) (1960). - Whey utilization. II Oxygen requirements of Saccharomyces fragilis growing in whey medium. Appl. Microbiol., 8, 291.

[47] Wasserman (A. E.) (1961). - Amino acid and vitamin composition of Saccharomyces fragilis grown in whey. J. Dairy Sci., 44, 379.

[48] Wasserman (A. E.), Hopkins (W. J.) and Porges (N.) (1958). - Whey utilization. I. Growth conditions for Saccharomyces fragilis. Sewage and Industrial Wastes, 30, 913. 
[49] Wasserman (A. E.), Hopkins (W. J.) and Porges (N.) (1959). - Rapid conversion of whey to yeast. XVth Intern. Dairy Congr., 2, 1241.

[50] Wasserman (A. E.) and Hampson (J. W.) (1960). - Whey utilization. III. Oxygen absorption rates and the growth of Saccharomyces fragilis in several propagators. Appl. Microbiol., 8, 293.

[51] Wasserman (A. E.), Hampson (J. W.), Alvare (N. F.) and Alvare (N. J.) (1961). - Whey utilization. V. Growth of Saccharomyces fragilis in a pilot plant. J. Dairy Sci., 44, 387.

[52] Wickerham (L. J.) (1946). - A critical evaluation of the nitrogen assimilation tests commonly used in the classification of yeasts. J. Bact., 52, 293.

[53] Wickerham (L. J.) and Duprat (E.) (1945). - A remarkable fission yeast, Saccharomyces versatilis nov. sp. J. Bact., 50, 597.

[54] Wickerham (L. J.) and Burton (K. A.) (1948). - Carbon assimilation tests for the classification of yeasts. J. Bact., 56, 363.

[55] Wickerham (L. J.) and Burton (K. A.) (1956). - Hybridization studies involving Saccharomyces lactis and Zygosaccharomyces ashbyi. J. Bact., 71, 290.

[56] Zuraw (E.) and Morgan (M. E.) (1952). - Acetaldehyde production by Streptococcus lactis and Streptococcus lactis var. maltigenes. (Abst.). J. Dairy Sci., 35, 483. 